\title{
Polypropylene-based long-life insecticide-treated mosquito netting
}

\author{
Walter W. Focke ${ }^{1, a, *}$ and Walter van Pareen ${ }^{2}$ \\ ${ }^{1}$ Department of Chemical Engineering, Institute of Applied \\ Materials, University of Pretoria, Lynwood Road, Pretoria \\ 0001, South Africa, e-mail: walter.focke@up.ac.za \\ ${ }^{2}$ Sasol ChemCity, 1 Sturdee Avenue, Rosebank, \\ Johannesburg, South Africa \\ *Corresponding author
}

\begin{abstract}
The World Health Organization (WHO) is promoting the use of insecticide treated nets (ITNs) in order to roll back malaria. Long-life netting (LLIN) is preferred and this study investigated the possibility of incorporating the insecticide in the fiber polymer. Multifilament polypropylene (PP) yarn, containing up to $0.76 \%$ of pyrethroid insecticide, was successfully produced on a conventional production-scale fiber-spinning machine. The insecticides were incorporated via masterbatches that contained up to $18.8 \%$ active with low-density polyethylene (LDPE) as the carrier. Nets knitted from the yarns were tested using bioassays with mosquitoes. Initial efficacy complied with WHO specifications but the performance deteriorated with the number of wash cycles. Crystalline insecticides, featuring a melting point above the wash temperature of $60^{\circ} \mathrm{C}$ (e.g., $\beta$-cyfluthrin), provided better wash resistance than amorphous insecticides (e.g., cyfluthrin).
\end{abstract}

Keywords: blooming; fiber spinning; insecticide; malaria; polypropylene.

\section{Introduction}

Malaria causes almost one million deaths a year and more than 300 million cases of severe illness [1]. The World Health Organization (WHO) regards insecticide treated nets (ITNs) as an important component in its efforts to roll back malaria. Extensive trials have established the efficacy of such nets to protect against a range of vector-borne diseases [2-11]. Current efforts focus on making such nets widely available and to popularize their use.

Initial development work concentrated on nets (periodically) treated with insecticides by a dipping process. However, in Africa the following problems were encountered:

- Low net retreatment rates because users did not perceive the need for it or the dipping procedure was deemed too complicated

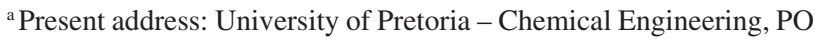
Box 35285 Menlo Park, 0102 Pretoria, South Africa.
- Insecticides were either not readily available or the local population considered them to be too expensive

- Treatment centers were not operational or were unsustainable.

It is preferable to treat nets at the factory level which would be restricted to professionals and the environmental impact of effluents can be better controlled. Netting materials used so far include cotton, nylon, polyester, polyethylene and polypropylene (PP). Multifilament netting is comfortable and performed better in ITN trials. In Africa, where transmission is intense, it was shown that it is not so much the net by itself but the insecticide on the net that provided protection against malaria vectors. Thus, it is vital that to ensure that the net is treated with an effective insecticide. Therefore, this study focused on long-life nets (LLINs) with the insecticide incorporated into the fiber polymer. A key target for WHO is a product that can resist more than 20 ISO normalized washes at $60^{\circ} \mathrm{C}[12,13]$.

Companies like BASF, Sumitomo and Vestergaard Frandsen have commercialized LLINs under the trade names Interceptor, Olyset and Permanet, respectively [9-11]. These nets feature improved wash and wear resistance and are recommended by WHO. Sumitomo Corporation's long life product, Olyset ${ }^{\mathrm{TM}}$, is based on high-density polyethylene monofilament fibers. This 56 mesh net weighs $50 \mathrm{~g} / \mathrm{m}^{2}$. The polymer contains $2 \%$ permethrin as the active insecticide. Field trials have shown that the initial insecticidal activity of this product is similar to that of ITNs with the same dosage but treated by the conventional dipping procedure.

Complex issues, such as safety, efficacy, lifespan and cost affect insecticide selection, formulation design and dosage level $[4,14,15]$. Synthetic pyrethroids are the only insecticides currently used in treated nets. WHO sanctions the use of a selected set of these insecticides [16]. The pyrethroids are lipophilic compounds that easily pass through cell membranes. They are absorbed through the skin, by inhalation or by ingestion. Their toxicity derives from an affinity for receptors or targets within the sodium channels essential for nerve conduction. Fortunately, their rapid metabolism greatly lowers the magnitude of the resultant toxicity.

Pyrethroid pesticides combine broad-spectrum insecticidal activity with low mammalian toxicity. Their short persistence in the environment is a desirable property. The pyrethroids declared acceptable to WHO for ITNs showed no teratogenic, carcinogenic or mutagenic effects in experimental toxicity studies. Low dosages are used for treating nets. They bind strongly to fabrics and they have a low volatility. The risk of oral and inhalation toxicity by the users of treated nets is therefore remote [6]. 
The ultimate purpose of this project was the development of long-lasting, PP-based mosquito netting conforming to WHO specifications. Here, we report on the properties of LLINs based on multifilament PP yarn with insecticides incorporated into the fiber polymer.

\section{Experimental}

\subsection{Materials}

Low-density polyethylene (LDPE) (Sasol grade LT 019/08 powder) was used as a carrier polymer for the insecticide masterbatches. PP fibers were spun using fiber grade HSV 103 ex Sasol Polymers, Johannesburg, South Africa (density $0.91 \mathrm{~g} /$ $\mathrm{cm}^{3}$ and Melt Flow Index (MFI) of $26 \mathrm{~g} / 10 \mathrm{~min} @ 230^{\circ} \mathrm{C}$ and $2.16 \mathrm{~kg}$ ). Table 1 lists the technical grade insecticides used in this study. There is a key difference between cyfluthrin and the other insecticides, and specifically $\beta$-cyfluthrin. At room temperature, cyfluthrin is a viscous liquid because it comprises a mixture of isomers while the others are crystalline solids.

\subsection{Characterization}

The relative volatility of the insecticides was determined with thermogravimetric analysis (TGA) performed on a Mettler Toledo STAR ${ }^{\text {e }}$ System. Samples weighing ca. $30 \mathrm{mg}$ were placed in open $70 \mu \mathrm{l}$ alumina pans. They were consolidated at the bottom of the cup by a melting-cooling cycle, by scanning the temperature from $25^{\circ} \mathrm{C}$ to $120^{\circ} \mathrm{C}$ and then back to $25^{\circ} \mathrm{C}$ at $20^{\circ} \mathrm{C} / \mathrm{min}$. This was necessary in order to allow comparison of evaporative mass loss rates. Data were collected during a subsequent heating run from $25^{\circ} \mathrm{C}$ to $300^{\circ} \mathrm{C}$ at a scan rate of $2^{\circ} \mathrm{C} / \mathrm{min}$. The TGA cell was purged with nitrogen flowing at a rate of $50 \mathrm{ml} / \mathrm{min}$ throughout the experiment.

Melting and crystallization behavior of the insecticides and the fiber polymer was studied by differential scanning calorimetry (DSC) on a Perkin-Elmer DSC 7 instrument. The temperature was scanned at $20^{\circ} \mathrm{C} / \mathrm{min}$ in nitrogen flowing at $50 \mathrm{ml} / \mathrm{min}$.

A small piece of yarn was placed onto carbon tape on a metal sample holder. It was coated with gold under argon gas using an autocoating unit E5200 (Polaron equipment LTD). The fibers were viewed on a JEOL 840 scanning electron microscope (SEM) under low magnification.
The spatial distribution of cyfluthrin in the fibers was investigated by confocal laser scanning microscopy on a Zeiss 510 META instrument (Carl Zeiss, Jena, Germany). It employed a Plan Apochromat 20X objective, NA=0.75 and a laser with a wavelength of $405 \mathrm{~nm}$.

\subsection{Compounding}

The masterbatching procedure was as follows. The powders were first mixed together in a tumbling drum before they were compounded on a 40-mm Berstorff $42 \mathrm{~L} / \mathrm{D}$ twin-screw machine fitted with two kneader block mixing sections. A flat temperature profile of $160^{\circ} \mathrm{C}$ was used. The extruded strands were cooled by passing them through a water bath before pelletizing. The initial target was to produce masterbatches containing $20 \%$ active. This proved difficult, especially for the two cyfluthrin actives. Above about $12 \%$, the solubility limit in the melt was exceeded, resulting in a two-phase system that prevented the formation of polymer strands that could be pulled through the water bath. In the end, a deltamethrin concentrate containing $18.8 \%$ active was successfully compounded. However, in order to avoid such problems with the other actives, the insecticide content of the other masterbatches was reduced to $10 \%$.

\subsection{Fiber spinning and net knitting.}

Multifilament yarn (110 dtex/32 fibers) was produced on a Tessilmeccanica Saronno Srl. Model FZ 8 Type FDY (1996). The inlet section, where the pellets were fed from the hopper into the extruder, was water-cooled. Three temperature controllers serviced the rest of the extruder and a further two, the jacket and fiber spinning head. The temperature profile was: $220^{\circ} \mathrm{C} / 240^{\circ} \mathrm{C} / 240^{\circ} \mathrm{C} / 240^{\circ} \mathrm{C} / 245^{\circ} \mathrm{C}$. The extruder screw speed varied from $490 \mathrm{rpm}$ to $524 \mathrm{rpm}$ during the trials. The draw ratio was set at 2.37 for most experiments but in some cases, it was necessary to adjust it upward (up to 2.97) to enable stable yarn production at the correct denier. Masterbatch was fed via a McGuire gravimetric feeder. Fasavin CP 45 (ex Chemische Fabriken Zschimmer \& Schwarz, Lahnstein, Germany) spin finish was dosed at ca. $1.5 \%$.

The masterbatch let down ratio determined the concentration of the insecticide in the final yarns. Table 2 lists the actives content of the yarns produced. It shows that, for deltamethrin, the concentrations investigated varied from $0.19 \%$ to $0.72 \%$. The lowest value represents an insecticide dosage

Table 1 Insecticides, their suppliers and the WHO recommended dosages for ITNs.

\begin{tabular}{lllll}
\hline Insecticide & CAS Number & Grade & Manufacturer & Dosage $^{1}\left(\mathrm{mg} / \mathrm{m}^{2}\right)$ \\
\hline Alphacypermethrin & {$[67375-30-8]$} & Technical & Tagros, India & $20-40$ \\
Bifenthrin & {$[82657-04-3]$} & Technical & Arysta, South Africa & - \\
$\beta$-Cyfluthrin & {$[68359-37-5]$} & Technical 95\% & Bayer, South Africa & - \\
Cyfluthrin & {$[68359-37-5]$} & Technical & Bayer, South Africa & 50 \\
Deltamethrin & {$[52918-63-5]$} & Generic 98\% & Tagros, India & $15-25$ \\
Lamdacyhalothrin & {$[91465-08-6]$} & Technical & Tagros, India & $10-15$ \\
\hline
\end{tabular}

${ }^{1}$ WHO recommended dosage [16]. 
Table 2 Initial effectiveness against mosquitoes in WHO Cone tests.

\begin{tabular}{|c|c|c|c|c|}
\hline Active $^{1}$ & $\begin{array}{l}\text { Content } \\
\%\end{array}$ & $\begin{array}{l}\text { Knock down } \\
1 \mathrm{~h}\end{array}$ & $\begin{array}{l}\text { Mortality } \\
24 \mathrm{~h}\end{array}$ & Format \\
\hline Alphacypermethrin & 0.29 & 100 & 100 & Net \\
\hline Alphacypermethrin & 0.38 & 100 & 100 & Net \\
\hline Bifenthrin & 0.38 & 100 & 100 & Net \\
\hline Cyfluthrin & 0.38 & 100 & 100 & Net \\
\hline$\beta$-cyfluthrin & 0.20 & 100 & 100 & Sock \\
\hline$\beta$-cyfluthrin & 0.38 & 100 & 100 & Net \\
\hline Deltamethrin & 0.19 & 100 & 100 & Sock \\
\hline Deltamethrin & 0.46 & 100 & 100 & Sock \\
\hline Deltamethrin & 0.72 & 100 & 100 & Sock \\
\hline Lambdacyhalothrin & 0.20 & 98 & 100 & Sock \\
\hline Negative & - & 0 & 3 & Sock \\
\hline
\end{tabular}

${ }^{1}$ Notes: All concentrations are given on a mass basis. All yarn samples were prepared using a draw ratio of 2.37 .

of ca. $75 \mathrm{mg} / \mathrm{m}^{2}$ for netting with a weight of $40 \mathrm{~g} / \mathrm{m}^{2}$. Table 1 shows that this corresponds to three times the WHO recommended quantity (upper limit) for deltamethrin in ITNs. However, in ITNs, the insecticide is applied to the outside surface of the fiber, while in LLINs it is incorporated inside the fiber polymer. A higher dosage is required for the latter to ensure that a sufficient quantity of the contact poison will be present at the surface of the fiber.

The tensile properties of yarn samples were determined at $50 \mathrm{~mm} / \mathrm{min}$ on an Instron Model 4303 machine at ambient conditions. Nets were knitted on a commercial warp-knitting machine, but small net samples were made on a laboratorysize knitter. The target weight for both fabrics was $40 \mathrm{~g} / \mathrm{m}^{2}$.

\subsection{Bioassays}

The efficacy of the various insecticides was first checked using bioassays on the smaller knitted samples. Selected yarns containing alphacypermethrin, bifenthrin, cyfluthrin, and $\beta$-cyfluthrin were also converted into nets (Table 2). Bioassays tests were performed at the Medical Research Council (MRC) in Durban and at the South African Bureau of Standards (SABS) in Pretoria, according to the relevant WHO guidelines for laboratory and field testing of long lasting insecticidal mosquito nets $[12,13]$. The MRC used Anopheles arabiensis non-blood fed female mosquitoes.

The test species were exposed to the netting material for a period of $3 \mathrm{~min}$, under standard WHO cones. The mosquitoes were thereafter removed, and the number of knockdowns determined after $1 \mathrm{~h}$. Then, a nutrient solution was made accessible to the mosquitoes and after $24 \mathrm{~h}$, mortality was quantified. The trials included a negative control comprising an untreated material. WHO standards require $>95 \%$ knockdown after $60 \mathrm{~min}$ and more than $80 \%$ mortality after $24 \mathrm{~h}$.

The washing procedure used was based on the WHO method $[12,13]$. The net samples were placed in $11 \mathrm{Schott}$ (transparent glass) bottles. Savon de Marseille soap was used to wash the mosquito nets. The soap was grated and $2 \mathrm{~g}$ was dissolved in $500 \mathrm{ml}$ deionised water per bottle. One piece of treated netting was introduced into a Schott bottle and placed in a water bath at $30^{\circ} \mathrm{C}$. The bottles were placed on a tray and agitated at 155 strokes per min for $10 \mathrm{~min}$ in the water bath. Thereafter, the net pieces were rinsed twice in deionised water. Net pieces were washed at daily intervals unless otherwise indicated. Between washes, they were allowed to air dry while hanging from lines in the laboratory. They were then wrapped in aluminum foil and stored at a relative humidity of $70 \%( \pm 10 \%)$ until tests were performed.

\subsection{Active content}

The active content of selected nets was determined by the SABS In-House Method 043/2009. The pyrethroid content impregnated in polymer fiber was also determined. Recovery determinations were carried out by adding known amounts of the active, e.g., alphacypermethrin, at different levels to portions of the neat PP control net sample and analyzing these concurrently with the samples. The SABS determined the $\beta$-cyfluthrin content following a similar procedure using a modified and validated version of the CIPAC method for alphacypermethrin [17].

\section{Results}

\subsection{Thermal analysis}

TGA traces are shown in Figures 1 and 2 for the insecticides in neat and masterbatch form, i.e., trapped in a polyethylene matrix. The mass loss during the heating runs reflects the evaporation of the active into the nitrogen atmosphere. It provides an indication of the volatility at elevated temperatures. The TGA curves for lambdacyhalothrin and $\beta$-cyfluthrin are not shown because they virtually coincide with the curves for cyfluthrin and alphacypermethrin, respectively. This means that bifenthrin is the most volatile insecticide while alphacypermethrin and $\beta$-cyfluthrin are the least volatile. The

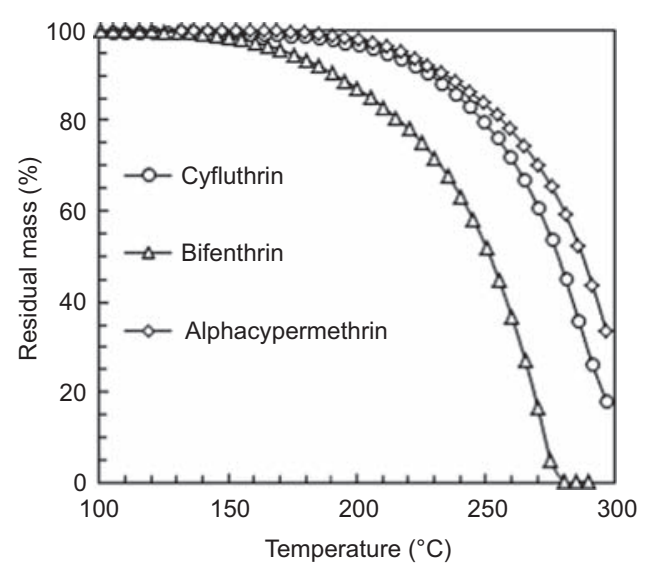

Figure 1 TGA traces obtained for selected neat additives. Temperature was scanned at $2^{\circ} \mathrm{C} / \mathrm{min}$ from 25 to $300^{\circ} \mathrm{C}$. 


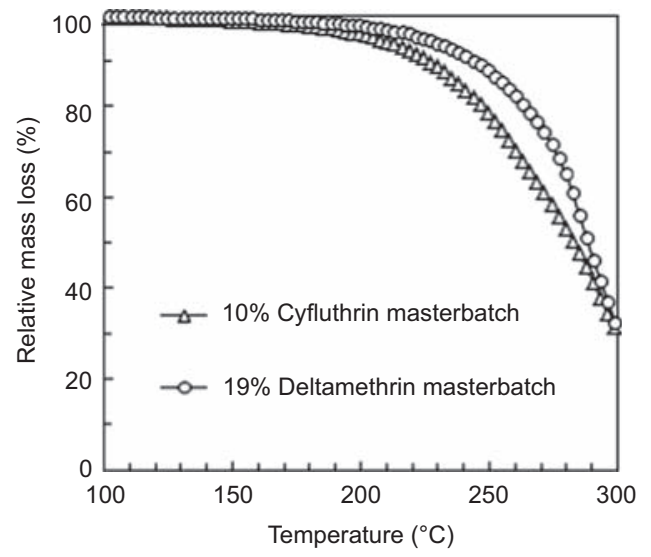

Figure 2 TGA traces obtained with the additives in masterbatch form with LDPE as carrier. The indicated mass loss was scaled with respect to the nominal active content.

neat insecticides show considerable volatility well below the polymer processing temperature of $245^{\circ} \mathrm{C}$. Comparison of the curves in Figures 1 and 2 shows that the volatility of the additives is somewhat suppressed when they are trapped in the polyethylene matrix. Considering their much lower concentration in the final yarn, this provides some hope that evaporative loss from the hot fiber formed during extrusion could be low.

Figure 3 shows the DSC curves for neat $\beta$-cyfluthrin. The traces obtained for deltamethrin, lambdacyhalothrin and alphacypermethrin showed similar behavior. All traces showed the expected endotherm peaks associated with the melting of the actives. However, no exothermic crystallization peaks were observed despite the fact that samples were cooled to $0^{\circ} \mathrm{C}$ (i.e., well below their melting points). The insecticides studied here did not crystallize easily from their respective melts. This contrasts with conventional crystalline organics that readily crystallize when cooled to below the melting temperature.

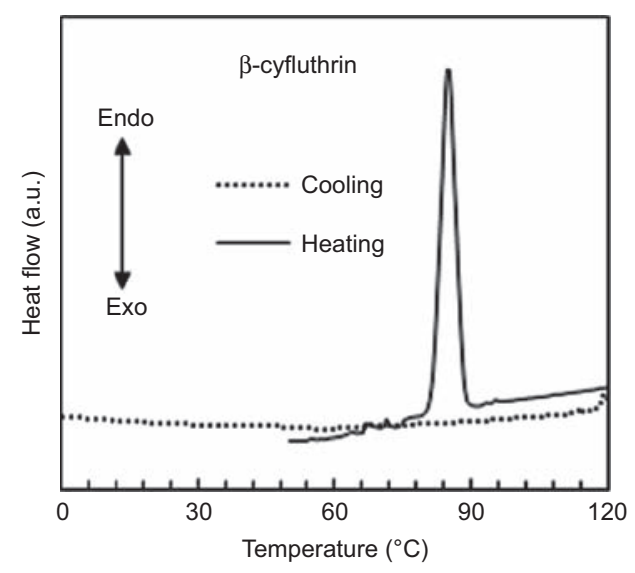

Figure 3 DSC traces obtained for $\beta$-cyfluthrin. Note the absence of a crystallization exotherm during the cooling run.
The rate of crystallization of the polymer is important during fiber spinning. It was therefore of interest to determine whether the presence of the masterbatch polymer and the insecticides might affect the crystallization behavior of the polypropylene. Figure 4 shows DSC cooling curves for polypropylene containing various levels of the LDPE. The shift of the exothermic crystallization peaks towards higher temperatures indicates that LDPE acts as a nucleating agent for polypropylene. The small endothermic peak, at ca. $90^{\circ} \mathrm{C}$ in the samples containing 5\% and 10\% LDPE, probably indicates crystallization of a polyethylene phase. Figure 5 shows the combined effect of insecticides and LDPE on the crystallization of PP during cooling runs in the DSC. It is clear that the insecticides, other than lambdacyhalothrin, inhibit the crystallization of the PP phase in the PP-LDPE blends. The net effect of the LDPE and insecticide combination is that the crystallization behavior is similar to that of the neat PP. This applies to the samples containing deltamethrin and $\beta$-cyfluthrin.

\subsection{Fiber spinning}

Fiber spinning trials proceeded smoothly. Fumes were liberated even during ordinary fiber spinning. However, as expected from the TGA results, fuming increased visibly when the yarn contained insecticides. Fume extraction was essential to ensure a safe working environment during trial runs.

\subsection{Yarn tensile properties}

The mechanical properties of the yarn are important in the knitting process and for the durability of the final net. Therefore, it was of interest to determine to what extent the incorporation of the insecticides and the polyethylene would affect the yarn's tensile properties. Neat PP yarn produced at a draw ratio of 2.37 had a tenacity of $3.6 \mathrm{~N} / \mathrm{dtex}$ and an elongation-to-break of $65 \%$. Inclusion of LDPE progressively reduces tenacity, and at $10 \%$, it reached a value of $2.1 \mathrm{~N} / \mathrm{dtex}$. Samples containing $2 \%$ or $5 \%$ LDPE showed higher elongation but at 10\% LDPE, it was similar to that of the neat PP.

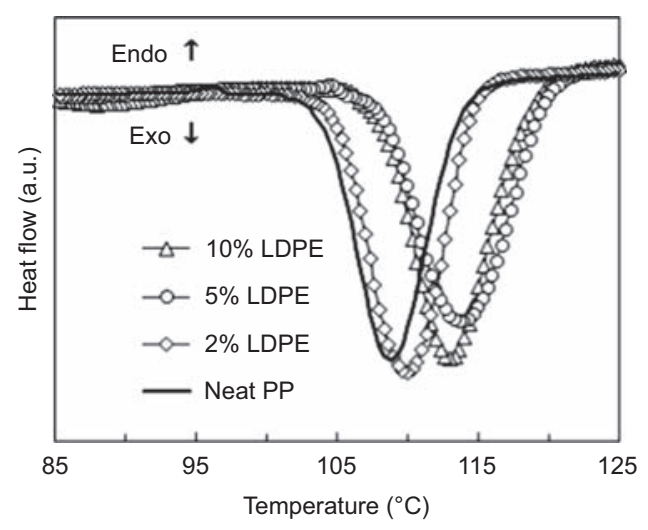

Figure 4 DSC cooling curves for PP containing different levels of LDPE. 


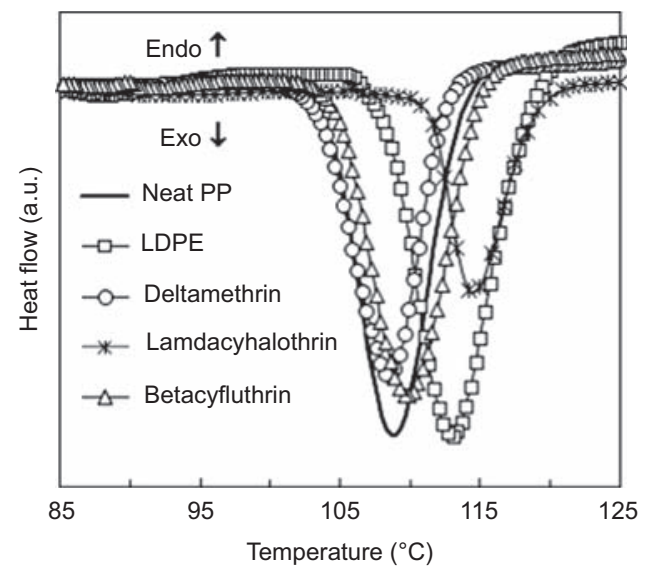

Figure 5 DSC cooling curves for PP with 10\% LDPE or 10\% of the active masterbatches added on.

Inclusion of the masterbatches also decreased the tenacity of the PP yarn and increased its elongation. Figures 6 and 7 present typical tenacity vs. strain curves. Increasing the insecticide masterbatch concentration caused a similar decrease in yarn tenacity and an increase in the elongation to break. For example, adding $4 \%$ of the $\beta$-cyfluthrin masterbatch decreased tenacity by about $30 \%$ but doubled the elongation to break. Note that the observed effects are attributable to the presence of both the LDPE and the insecticide. It should be possible to increase the tenacity by increasing the draw ratio. Trials using PP containing deltamethrin and/or LDPE confirmed this.

Figure 6 shows the effect of different additives together with the carrier polymer on the tenacity vs. strain curves. The tenacity-strain behavior of the lambdacyhalothrin yarn differed from that of the yarns containing alphacypermethrin or deltamethrin. The rate of crystallization during the dynamic fiber spinning process is an important factor affecting fiber

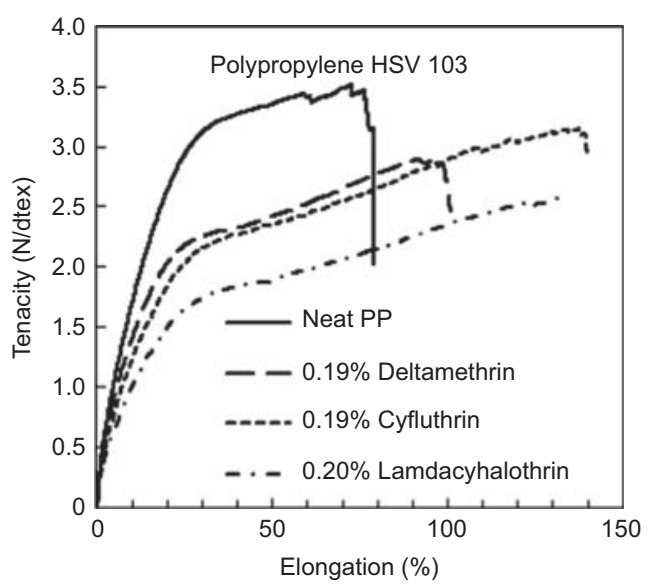

Figure 6 Representative stress-strain curves showing the effect of different additives together with the LDPE carrier polymer on the tensile properties of yarn produced at a draw ratio of 2.37 .

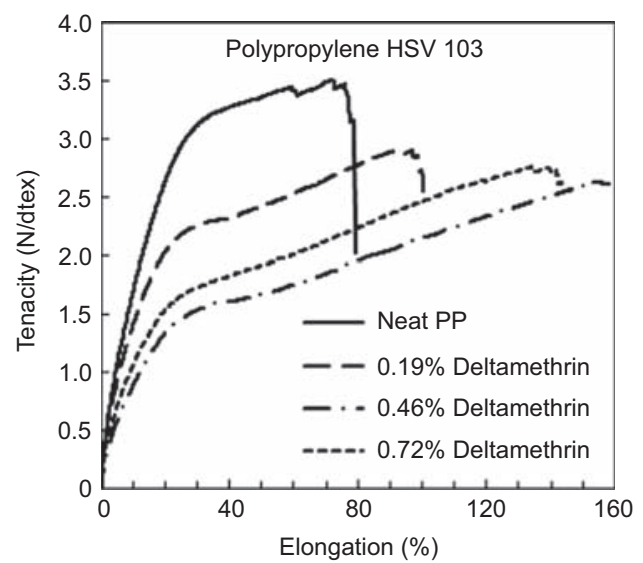

Figure 7 Representative stress-strain curves showing the effect of the additive together with the LDPE carrier polymer concentration on the tensile properties of yarn produced at a draw ratio of 2.37 .

morphology and ultimate properties. Figure 5 revealed that the behavior of lamdacyhalothrin differed from the others in that it had little effect on the rate of polypropylene crystallization. It is, therefore, not surprising that the final yarn properties differed too when this additive was present. It definitely reduced the tenacity and increased the yarn elongation more than the other two insecticides.

Figure 7 shows the effect of increasing the deltamethrin plus LDPE concentration on yarn mechanical properties. Interestingly, the trend towards lower tenacity and higher elongation is not regular. The reasons for this are not currently understood and will require investigation that is much more detailed.

These results indicate that the presence of insecticides and the inclusion of LDPE had a significant effect on the mechanical properties of the yarn. Nevertheless, it was possible to reliably knit nets using the machine settings optimized for the neat PP yarn. While the tensile strength generally decreased, the improvement in the elongation to break is actually good news, as it potentially provides the net with more "give" before it will tear.

\subsection{Initial effectiveness against mosquitoes}

Table 2 provides an overview of the bioassay results for unwashed insecticide-containing socks and nets. The WHO standards specify $\geq 95 \%$ knockdown after $1 \mathrm{~h}$ and $\geq 80 \%$ mortality after $24 \mathrm{~h}$. Samples containing any of the six insecticides tested conformed to this requirement at loading as low as ca. $0.2 \%$.

\subsection{Effect of washing on net performance}

Additives are lost from the fiber surface via sublimation, evaporation or mechanical scuffing. The former rates are very slow for the current set of pyrethroids. For net lifetimes $<5$ years, they can probably be neglected. However, the latter mechanism also operates when the net is washed. 
Current WHO standards for long lasting insecticide treated nets require that long lasting nets should retain their efficacy even after 20 standardized washes [12,13]. Every wash removes 30-95\% of insecticide deposited on the surface [5].

It is clear that the ability of multifilament yarn to retain efficacy beyond a few washes is of paramount importance. Figure 8 shows data for nets washed three times. Nets containing $0.38 \%$ cyfluthrin or $0.29 \%$ alphacypermethrin retained high bioactivity when tested 6 weeks later, but the net containing only $0.19 \%$ bifenthrin already failed WHO criteria. Unfortunately, the net containing $0.38 \%$ cyfluthrin failed the bioassay criterion after just five washes, with a knockdown of $7 \%$ and a mortality of only $13 \%$.

Figure 9 shows additional data for a net containing $0.38 \%$ alphacypermethrin. In this case, the nets were washed in 3-day intervals and tested immediately. Mortality decreased rapidly and performance was already marginal after a single wash. Knockdown activity was retained for longer, but the WHO criterion of $95 \%$ was failed after 10 washes.

Figure 10 shows much better performance of a net containing $0.38 \% \beta$-cyfluthrin. In this case, the net was washed every other day and the bioactivity determined using the WHO cone test. Performance complied with the WHO requirements even after 25 washes but they were not fulfilled after 30 washes.

Figure 11 shows the effect of washing on the residual active content of the nets. The nets initially contained a nominal concentration of $0.38 \%$ active each. The alphacypermethrin nets appear to suffer sustained loss of insecticide as the number of washes increases. This is also true for $\beta$-cyfluthrin, but for this net, the difference in active content after five and 20 washes is relatively small.

\section{Discussion}

The temperature and the fraction amorphous phase determine insecticide solubility in polymers. Solubility generally increases with temperature. Polymer additives may dissolve in the amorphous parts of polymer matrices but are insoluble in crystalline regions. This implies that the insecticide will

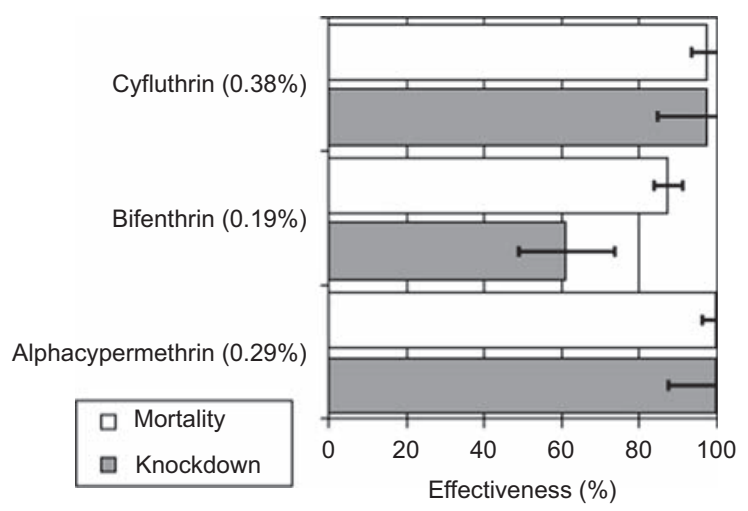

Figure 8 The bioactivity of nets that were washed three times and tested after 6 weeks. The error bars indicate one standard error above and below the mean values.

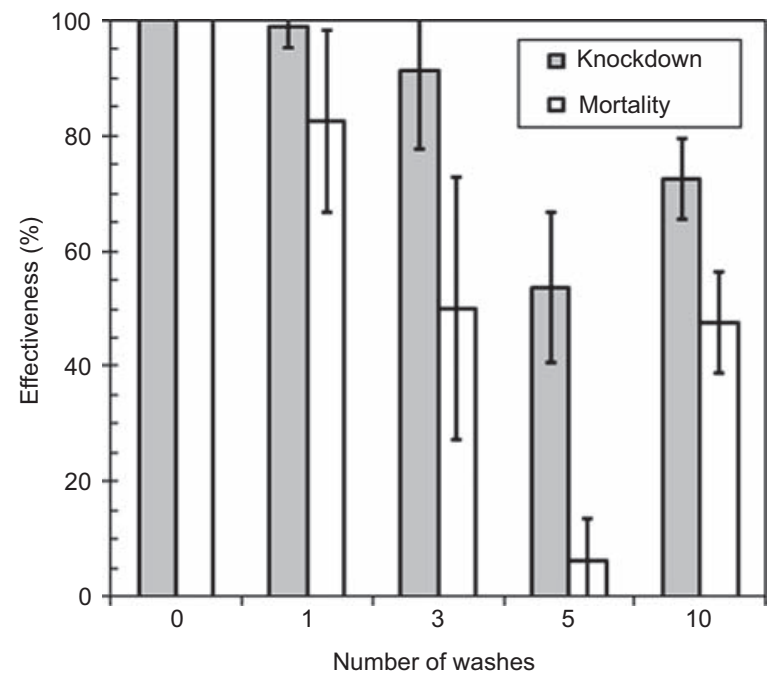

Figure 9 The effect of washing on a net containing $0.38 \%$ alphacypermethrin. The efficacy was determined 2 days after the last wash using the WHO cylinder test. The error bars indicate one standard error above and below the mean values.

have a higher solubility in the molten polymer than in the drawn fiber at ambient temperature. Full dissolution in the melt is advantageous as it aids homogeneous dispersion of the solute in the matrix.

During fiber spinning, the melt is cooled, stretched and drawn. The process involves controlled polymer crystallization. Concomitantly, the fraction amorphous phase, available to dissolve the additive, is diminished. In practice, the insecticide dosage level exceeds its solubility limit at ambient conditions and it may therefore become trapped in a supersaturated state inside the polymer. In an effort to return to

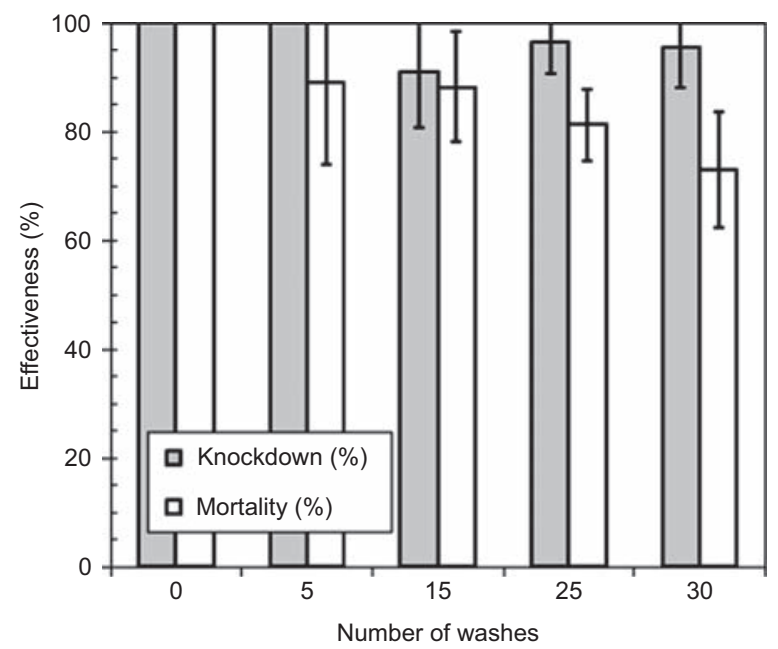

Figure 10 The effect of washing on a net containing $0.38 \%$ $\beta$-cyfluthrin. The nets were repeatedly washed on consecutive days and performance evaluated using the WHO cone test several days after the last wash. The error bars indicate one standard error above and below the mean values. 


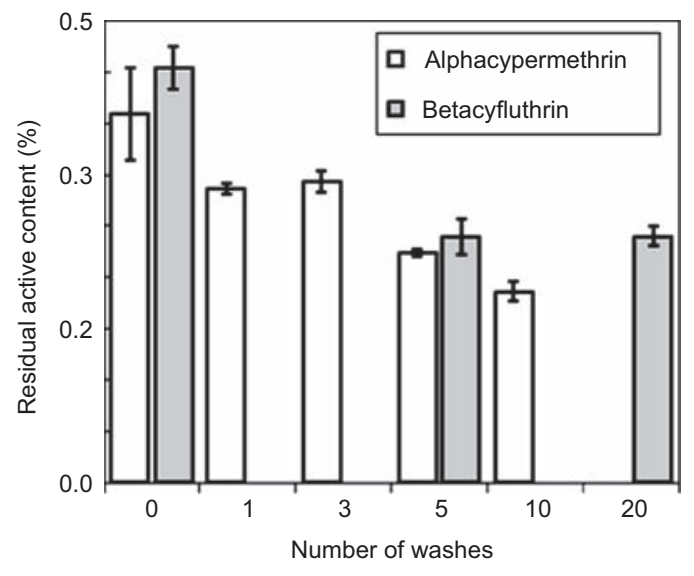

Figure 11 The effect of washing on the residual active content of nets containing nominally $0.38 \%$ alphacypermethrin or $\beta$-cyfluthrin, respectively. The reported values were corrected for recovery. The error bars indicate one standard error above and below the mean values.

an equilibrium state, the additive may diffuse to the surface of the fiber. Here, it accumulates and may eventually form crystalline deposits.

The DSC data indicated that the pyrethroid additives, at least in the early stages, stay amorphous when cooled rapidly from their melt. Figure 12 schematically illustrates the blooming process for an idealized amorphous additive present above its glass transition temperature. In this analysis, we assume that the fiber can be approximated by an infinite cylinder with isotropic properties in the hoop and radial directions. It is also assumed that, immediately after spinning the fiber, the active is homogeneously dispersed throughout the amorphous regions in the fiber (State $\mathrm{A}$ ). The concentration $C_{i}$ is determined by the dosage and exceeds the equilibrium concentration $C_{e q}$. The additive diffuses to the surface setting up a concentration profile inside the fiber (State B). Ultimately, after a sufficiently long time, the concentration in the fiber is

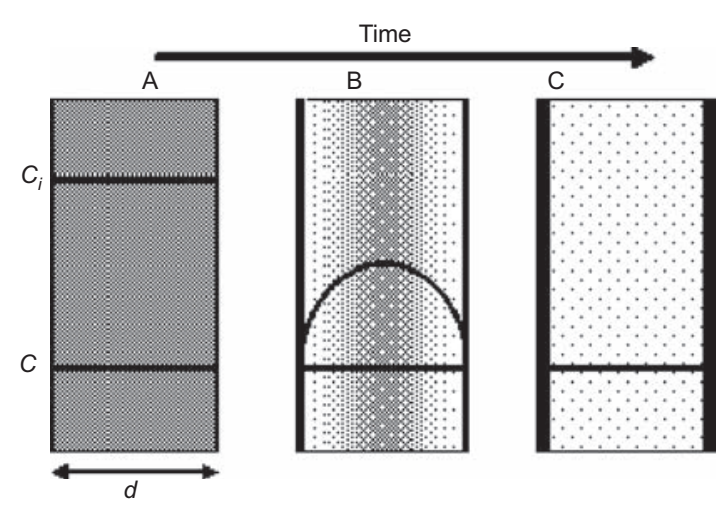

Figure 12 Schematic of the surface blooming mechanism in a fiber with diameter $d$. The intensity of the gray color scales with the insecticide concentration. reduced to a homogeneous concentration equal to the solubility limit $C_{e q}$ (State C).

Blooming is an undesirable phenomenon when the additive is a stabilizer, colorant, etc. [18-21]. It leads to the effective loss of active from the system and causes surface blights. The insecticides incorporated into the fiber polymer are contact poisons. To be effective, they have to be available above the minimum effective concentration on the fiber surface. Thus, in this application, blooming is a desirable process that facilitates additive transport to and accumulation at the fiber surface.

The amount of insecticide that dissolves in the bulk of the polymer matrix is retained there. This quantity is not available for the intended use, as it will not migrate to the fiber surface. Clearly, actives with low polymer solubility are preferred. Unfortunately, the solubility of the pyrethroids in polymers is unknown. Perusal of the technical literature indicated that the solubility range of typical commercial additives in PP is $0.005 \%<C_{e q}<2 \%$ [20-23].

The fiber geometry approximates to an infinite cylinder of diameter $d$. The additive concentration $C$ is described in molar terms with units, such as $\mathrm{mol} / \mathrm{m}^{3}$. However, in technological practice, the mass fraction (or percentage) is used. Since the solubility of the additive is very low, the two quantities are directly proportional. The depletion of the excess insecticide inside the fiber is described by the diffusion equation:

$\frac{\partial C}{\partial t}=D \frac{\partial^{2} C}{\partial r^{2}}$

Initial condition: $C=C_{i}$ at $t=0$

Boundary condition: $C=C_{e q}$ at $r=d / 2$

since heat and mass transport are analogous, solutions obtained for the equivalent heat transfer problem are applicable [24]. The analytical infinite series solution to Eq. (1), and its associated initial and boundary conditions, is inconvenient as it converges slowly. For "long times" (defined by the Fourier Number exceeding $F o=D t / d^{2}>0.1$ ) a useful approximate expression for the mean excess concentration in the fiber is [24]:

$\frac{\langle C\rangle-C_{e q}}{C_{i}-C_{e q}}=0,692 \exp \left(-23,13 \frac{D t}{d^{2}}\right)$

where $\langle C\rangle$ is the average concentration of active inside the fiber.

Unfortunately, the diffusion coefficients of the pyrethroids in PP are also unknown. However, typical diffusion coefficient values for commercial additives in PP range from ca. $\mathrm{D}=5.10^{-16}$ to $\mathrm{D}=5.10^{-12} \mathrm{~m}^{2} \cdot \mathrm{s}^{-1}[23,25-28]$. It is assumed that these values also span the likely diffusion coefficient values for the three pyrethroids at hand.

Eq. (4) was used to estimate the time to $95 \%$ depletion of the excess insecticide initially trapped in the fiber. The results are shown in Figure 13. It shows two solid lines calculated using the limiting diffusion coefficients given above. It is clear from Figure 13 that, for the current fiber 
diameter of $d=22 \mu \mathrm{m}$, blooming will be essentially complete within a single day. It can be assumed that washing effectively removes most of any amorphous active that is present on the surface of the fibers. The implication is that amorphous actives present above their glass transition temperature (e.g., cyfluthrin) are unlikely to provide sufficient wash resistance to be of practical use. Below, it is shown that there is circumstantial evidence that this is indeed the case for cyfluthrin.

The data presented above provide evidence that at least some nets retain their bioactivity for several wash cycles. It is therefore of interest to determine the actual distribution of the actives in the polymer fiber. Fortunately, some of the actives are fluorescent and this property was exploited with confocal microscopy. This technique facilitates three-dimensional (3-D) visualization of the additive distribution in the fiber. Figure 14 merely shows a 2-D representation of the distribution of cyfluthrin in an unwashed net. Visual inspection of the 3-D image revealed the presence of small fluorescent spots throughout the fiber with areas of higher intensity fluorescence near or at the surface of the fibers. The image obtained for the washed net made from the same yarn showed very weak fluorescence, suggesting that virtually all the active was lost after even five washes. In contrast, a net made from deltamethrin still showed comparable fluorescence even after 10 washes. This difference may be attributable to the difference in the physical nature of the two additives. Deltamethrin is highly crystalline with a high melting point $\left(<100^{\circ} \mathrm{C}\right)$ while cyfluthrin is a viscous liquid at room temperature, i.e., it is amorphous and above its glass transition temperature. It is therefore easier to remove the cyfluthrin from the fiber through a washing process than to remove deltamethrin or $\beta$-cyfluthrin once they are deposited as crystalline particles in the fiber.

The second assumption made above concerning the isotropic nature of the idealized fiber is also not valid. SEM pictures of the fibers showed the clear presence of a thin skin-like structure that covers the outside of the fibers. It appears that this skin is not strongly bonded to the fiber

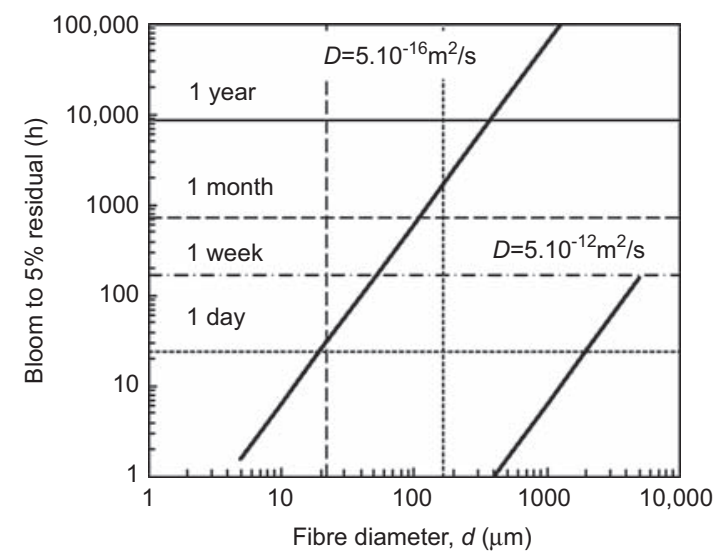

Figure 13 Effect of fiber diameter on the time to bloom down to $5 \%$ residual additive as predicted by Eq. (4).

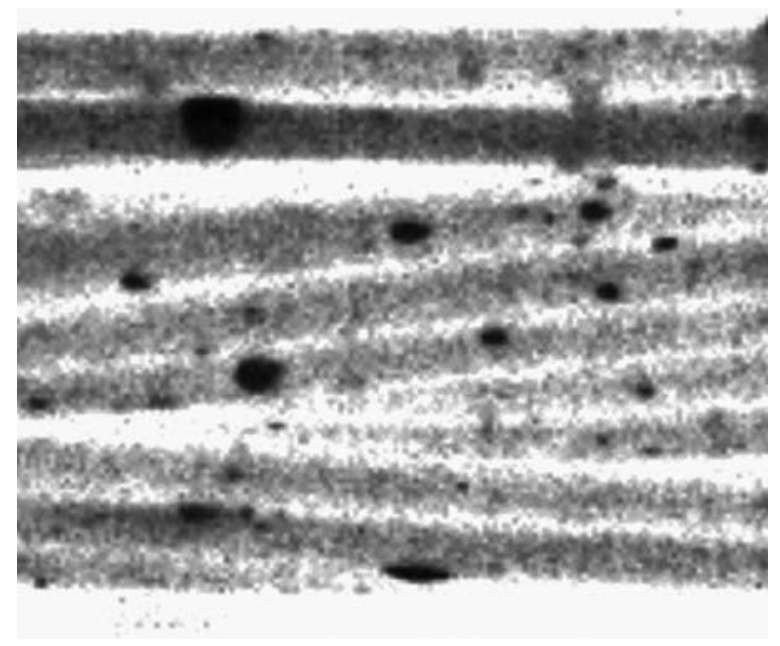

Figure 14 Confocal microscope image of a bundle of 10 horizontal fibers containing $0.38 \%$ cyfluthrin. The luminescence is shown in negative gray scale so that darker regions represent higher the concentrations of the active. Noteworthy is the uneven distribution of the cyfluthrin trapped inside the fibers and the presence of insecticide spots on or close to the fiber surface.

core. It easily detaches to form blisters when the fiber is severely mechanically stressed. Figure 15 shows a particularly striking picture of such a piece of skin folded back over the fiber shaft and revealing the structure underneath. Confocal microscopy revealed an intense fluorescence from the underside of this skin. This suggests that the skin may represent a membrane-like barrier to outward migration of the actives. Thus, active may be accumulating just below this skin rather than on the outside of the fiber. Clearly, if this were the case, better wash resistance would be the result. While the skin morphology was also observed in neat PP fibers, SEM pictures indicated that it was more noticeable in the fibers containing LDPE.

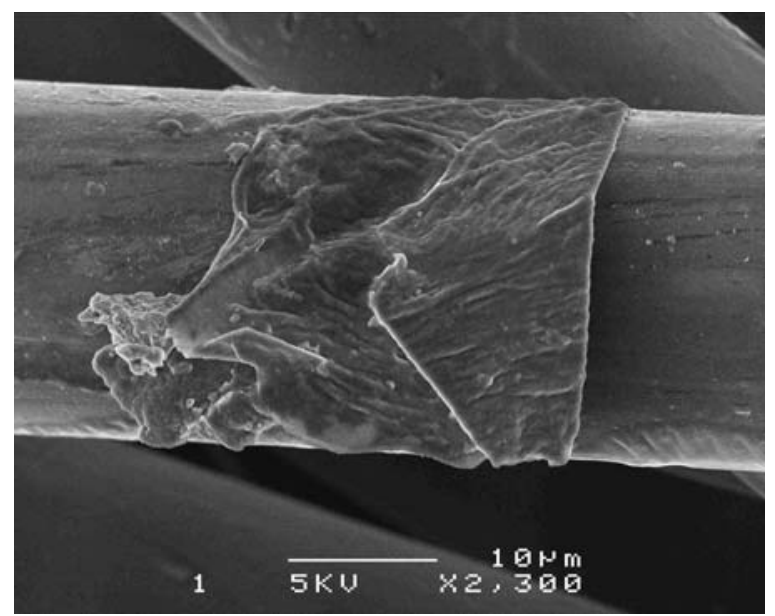

Figure 15 SEM picture of a damaged part of a fiber containing $0.57 \%$ cyfluthrin showing the covering "skin". 


\section{Conclusions and recommendations}

Multifilament PP yarns containing WHO approved insecticides were successfully produced on a production spinning line. The fume generation during extrusion with pyrethroids necessitates superior ventilation, extraction and scrubbing systems.

The tenacity of the PP yarn prepared at a draw ratio of 2.37 was about $3.6 \mathrm{~N} / \mathrm{dtex}$ and the elongation about 60-90\%. The incorporation of actives via LDPE masterbatches lowered the tenacity of the yarns by up to $35 \%$ and increased the elongation up to a factor of two. These dramatic property changes did not have a detrimental effect on knitting behavior.

Efficacy testing results for unwashed nets showed encouraging results. Most samples containing $\geq 0.19 \%$ active complied with the WHO requirements ( $>95 \%$ knockdown after 60 min and $>80 \%$ mortality after $24 \mathrm{~h}$ ). Analysis reveals that blooming from the thin fibers in the multifilament yarn will be rapid with a time constant less than one day. Washing is known to remove in excess of $30 \%$ active on the fiber surface per wash. This implies that it is unlikely that fibers containing amorphous additives, such as cyfluthrin will achieve the minimum of 20 washes stipulated by WHO. Better results were obtained with the crystalline $\beta$-cyfluthrin form. The implication is that only crystalline actives with melting points well above the wash temperature hold promise for good wash resistance.

\section{Acknowledgements}

The authors express their gratitude to Sasol ChemCity for financial support for this project, and to Mr Alan Hall of the Centre for Microscopy for performing the SEM and confocal microscopy work.

\section{References}

[1] Kokwaro G. Malaria J. 2009, 8 (Suppl. 1), art. no. S2.

[2] Lines JD, Myamba J, Curtis CF. Med. Vet. Entomol. 1987, 1, 37-51.

[3] Njunwa KJ, Lines JD, Magesa SM, Mnzava AEP, Wilkes TJ, Alilio M, Kivumbi K, Curtis CF. Acta Tropica. 1991, 49, 87-96.

[4] Curtis CF, Wilkes TJ, Myamba J, Chambika C. Trans. R. Soc. Trop. Med. Hyg. 1994, 88, 373.

[5] Miller JE, Jones CO, Ndunguru S, Curtis V, Lines J. Trop. Med. \& Intl. Health 1999, 4, 167-174.
[6] Zaim M, Aitio A, Nakashima N. Med. Vet. Entomol. 2000, 14, $1-5$.

[7] Curtis C. Outlook. Pest Man. 2005, 16, 50-51.

[8] Teklehaimanot A, Sachs JD, Curtis C. Lancet 2007, 369, 2143-2146.

[9] Malima RC, Magesa SM, Tungu PK, Mwingira V, Magogo FS, Sudi W, Mosha FW, Rowland M. Malaria J. 2008, 7, art. no. 38.

[10] Kitua AY, Mboera L, Magesa SM, Maxwell CA, Curtis CF. Science 2008, 319 (5865), 900-900.

[11] Banek K, Kilian A, Allan R. Malaria J. 2010, 9, art. no. 84.

[12] WHO/CDS/WHOPES/GCDPP/2005.11 Guidelines for laboratory and field testing of long lasting insecticidal mosquito nets. http://whqlibdoc.who.int/hq/2005/WHO_CDS_WHOPES_ GCDPP_2005.11.pdf. Accessed 16 September 2011.

[13] WHO/CDS/NTD/WHOPES/GCDPP/2006.3 World Health Organization: guidelines for testing mosquito adulticides for indoor residual spraying and treatment of mosquito nets. http:// whqlibdoc.who.int/hq/2006/WHO_CDS_NTD_WHOPES_ GCDPP_2006.3_eng.pdf. Accessed 16 September 2011.

[14] Gonzales AP, Licastro SA, Zerba E. Pestic. Sci. 1999, 55, $1187-1193$.

[15] Gonzales AP, Licastro SA, Zerba E. Pestic. Manag. Sci. 2001, $58,183-189$.

[16] WHO recommended insecticide products treatment of mosquito nets for malaria vector control. http://www.who.int/whopes/ Insecticides_ITN_Malaria_ok3.pdf. Accessed 16 September 2011.

[17] Alpha-Cypermethrin 454, CIPAC 4508/R, method extension for LN. http://www.cipac.org/document/prepublished_methods/ alpha\%20cypermethrin\%20LN.pdf. Accessed 14 September 2011.

[18] Bair HE. Pol. Eng. Sci. 1973, 13, 435-439.

[19] Calvert PD, Billingham NC. J. Appl. Pol. Sci. 1979, 24, 357-370.

[20] Billinghham NC. Degradation and stabilization of polymers. Chap. 10. In Materials Science and Technology, Cahn RW, Haasen H, Kramer EJ, Eds., Wiley-VCH: Weinheim, 2000.

[21] Ibe EC. J. Appl. Pol. Sci. 1970, 14, 837-846.

[22] Mar'in AP, Borzatta V, Bonora M, Greci L. J. Appl. Pol. Sci. 2000, 75, 883-889.

[23] Foldes E. J. Appl. Pol. Sci. 1993, 48, 1905-1913.

[24] Beek WJ, Mutzall KMK, van Heuven JW. Transport Phenomena, Wiley \& Sons: Chichester, 1999.

[25] Limm W, Hollifield HC. Food Addit. Contam. 1996, 13, 949-967.

[26] Mar'in AP, Borzatta V, Bonora M, Greci L. J. Appl. Pol. Sci. 2000, 75, 897-903.

[27] Mercea P. In Plastics Packaging Materials for Food. Barrier Function, Mass Transport, Quality Assurance and Legislation, Pirringer O-G, Baner AL, Eds., Weinheim: New York, 2000.

[28] Reymier CR, Dole P, Feigenbaum A. J. Appl. Pol. Sci. 2001, 82, 2434-2443. 